\title{
Avaliação do teor de elementos químicos em manguezais da ilha de Itaparica, Bahia, Brasil
}

\author{
Leila Maria Mendes Santosa, Rosemário Cequeira Souzab, Daniela Santos Anunciaçãoc, Icaro Thiago Andrade \\ Moreira $^{d}$, Vera Lúcia Câncio Souza Santosa,b, Zenira Cardoso Vilasboas Viana ${ }^{\mathrm{e}}$ \\ a Programa de Pós-Graduação em Geoquímica: Petróleo e Meio Ambiente, Universidade Federal da Bahia, Salvador, 40170-290, Bahia, Brasil. \\ ${ }^{\mathrm{b}}$ Departamento de Quimica Analítica, Universidade Federal da Bahia, Salvador, 40.110-100, Bahia, Brasil. \\ ${ }^{\mathrm{c}}$ Instituto de Química e Biotecnologia, Universidade Federal de Alagoas, Maceió, 57072-900, Alagoas, Brasil. \\ d Núcleo de Estudos Ambientais, Universidade Federal da Bahia, Salvador, 40170-290, Brasil. \\ e Departamento de Bioquímica e Biofísica, Universidade Federal da Bahia, Salvador, 40170-290, Brasil. *zenira@ufba.br
}

Recebido: 31 setembro 2017 / Aceito: 28 dezembro 2017 / Publicado online: 20 janeiro 2018

\begin{abstract}
Resumo
Os teores de $\mathrm{Fe}, \mathrm{Mn}, \mathrm{Mo}, \mathrm{Ni}, \mathrm{Cu}, \mathrm{Ba}$ e V são relatados em sedimentos superficiais dos manguezais de sete povoados: Baiacu, Cacha Pregos, Jeribatuba, Misericórdia, Mocambo, Campinas e Ponta Grossa. Todos eles da Ilha de Itaparica, localizada na Baía de Todos os Santos (Bahia). Valores equivalentes ao percentual de matéria orgânica e de carbonato de cálcio e da análise granulométrica também são apresentados nas amostras investigadas. Os elementos traço e majoritários foram determinados por Espectrometria de Emissão Ótica com Plasma Indutivamente Acoplado (ICP OES) e as maiores concentrações $\left(\mu \mathrm{g} \mathrm{g}^{-1}\right)$ foram encontradas principalmente nas regiões de Misericórdia (Mn: 36,35 $\pm 1,14$; Ba: 11,01 $\pm 1,71$; V: 10,55 $\pm 1,81$; Mo: 10,77 $\pm 1,82$ ), Cacha Pregos (Mn: 26,75 \pm 2,51; Ba: 9,77 \pm 1,42; Cu: 20,38 $\pm 6,55$ ) e Jeribatuba (Ba: 9,88 $\pm 0,46$ ). Concentrações elevadas de Mn em Baiacu e Ponta Grossa também foram apresentadas. Análises estatísticas expressas por Matriz de Correlação de Pearson e Análise de Componentes Principais (PCA) estabeleceram relações entre as variáveis e amostras estudadas, corroborando as concentrações de metais encontradas nas amostras de Misericórdia, Cacha Pregos e Jeribatuba.
\end{abstract}

Palavras-chave: ICP OES, matéria orgânica, metais, poluição, sedimentos

\section{Evaluation of chemical elements content in mangroves island of Itaparica, Bahia, Brazil}

\begin{abstract}
The content of $\mathrm{Fe}, \mathrm{Mn}, \mathrm{Mo}, \mathrm{Ni}, \mathrm{Cu}, \mathrm{Ba}$ and $\mathrm{V}$ are reported in surface sediments of mangrove of seven settlements: Baiacu, Cacha Pregos, Jeribatuba, Misericórdia, Mocambo, Campinas e Ponta Grossa. All of them from the island of Itaparica, Bahia. Values equivalent to the percentage of organic matter and calcium carbonate and particle size analysis are also presented in the samples investigated. Trace and major elements were determined by ICP OES and higher concentrations $\left(\mu \mathrm{g} \mathrm{g}^{-1}\right)$ were found mainly in the Misericórdia (Mn: $36.35 \pm 1.14$; Ba: $11.01 \pm 1.71$; V: $10.55 \pm 1.81$; Mo: $10.77 \pm 1.82$ ) . Cacha Pregos (Mn: 26.75 \pm 2.51 ; Ba: $9.77 \pm 1.42$; $\mathrm{Cu}: 20.38 \pm 6.55$ ) and Jeribatuba (Ba: $9.88 \pm 0.46$ ). High concentrations of Mn in Baiacu and Ponta Grossa were also presented. Statistical analysis expressed by Pearson Correlation Matrix and Principal Component Analysis (PCA) established relationships between variables and samples studied, corroborating metal concentrations found in the samples of Misericórdia, Cacha Pregos and Jeribatuba.
\end{abstract}

Keywords: ICP OES, metal, organic matter, pollution, sediment

\section{Introdução}

Alguns elementos químicos são considerados como contaminantes globais, devido à elevada toxicidade, propriedades não biodegradáveis, ação acumulativa, além da associação de alguns destes a diversas fontes de contaminação (Carapeto, 2008). Pesquisas indicam que o sedimento se comporta como principal suporte na deposição de elementos químicos.
Neste contexto, encontram-se os manguezais, ecossistemas constituídos de sedimentos, água, vegetação e organismos que ficam predispostos à exposição de diversos elementos químicos com ações persistentes e atividades biogeoquímicas (Queiroz e Celino, 2008; Gan et al., 2013).

A decomposição da matéria orgânica (MO), presença de partículas finas (silte e argila) e hidróxidos de ferro e magnésio são fatores que podem explicar a acumulação destes elementos 
nos substratos de manguezal. Além disso, materiais calcários, constituídos principalmente de esqueletos de corais e carbonato de cálcio de origem orgânica, como carapaças e conchas, são observados neste ecossistema. (Castro e Huber, 2012).

A depender das condições de $\mathrm{pH}$ do meio, os materiais calcáreos formam compostos pouco solúveis com $\mathrm{Cu}, \mathrm{Zn}$ e $\mathrm{Pb}$ (Fortunato, 2009; Boaventura, 2011) podendo haver a incorporação dos metais pela biota local, que por sua vez podem conduzir a efeitos tóxicos com tendências a bioacumulação ao longo da cadeia alimentar. (Dou et al., 2013).

A ilha de Itaparica, na Bahia, apresenta uma série de manguezais dispostos ao longo de sua porção ocidental, localizados em regiões, dentre as quais se destacam os municípios de Baiacu (BA), Cacha Pregos (CP), Jeribatuba (JE), Misericórdia (MI), Mocambo (MU), Campinas (CA) e Ponta Grossa (PG). Estes manguezais têm sido submetidos a muitos impactos ambientais, decorrentes da ação antrópica, como derramamento de petróleos e descarte de efluentes (Martins et al., 2015).

A importância do desenvolvimento de estudos desta natureza se justifica pelas atividades econômicas desenvolvidas nos manguezais, como pesca e mariscagem, e também do ponto de vista social, aonde muitas famílias pescam e mariscam espécies presentes nos manguezais.

Este consumo contínuo, a depender do tipo de elemento químico e da concentração presente no sedimento que será disponibilizada para os organismos locais, pode promover a acumulação de elevadas concentrações ao longo da cadeia trófica que por sua vez poderá alterar a qualidade de vida dos indivíduos que consomem estes alimentos.

Esta pesquisa apresenta um estudo, avaliando as condições ambientais e contribuindo com informações que possam prevenir ou reduzir impactos negativos nestes ecossistemas. Com esse propósito, foi realizada a investigação do teor de metais $(\mathrm{Cu}, \mathrm{Ba}, \mathrm{Mo}, \mathrm{V}, \mathrm{Fe}, \mathrm{Ni}$ e $\mathrm{Mn})$ presentes em amostras de sedimentos de diversos manguezais na Ilha de Itaparica, estabelecendo, através de análises estatísticas, uma relação com a granulometria dos sedimentos, com a presença da matéria orgânica (MO) e carbonatos.

\section{Material e Métodos}

\section{Área de amostragem}

Vinte e oito provas de sedimentos superficiais $(0-5 \mathrm{~cm}) \mathrm{de}$ manguezais foram coletadas, no mês de dezembro de 2010, em sete regiões da Ilha de Itaparica, conforme expresso na Figura 1, utilizando-se um sistema de posicionamento global (GPS) para mapear a posição de cada ponto de amostragem. As coletas foram realizadas nos povoados de BA, CP, JE, MI, MU, CA e PG. Para cada região, foram selecionados 4 pontos, em quadrados de $90 \times 90 \mathrm{~cm}$, a cada $10 \mathrm{~m}$, totalizando 4 amostras homogeneizadas de aproximadamente $150 \mathrm{~g}$.

\section{Metodologia utilizada}

A análise granulométrica foi realizada pesando-se aproximadamente $12 \mathrm{~g}$ da amostra, em um cadinho e aquecimento na mufla a $500^{\circ} \mathrm{C}$ por $5 \mathrm{~h}$ para decomposição da MO, até peso constante. Após isso, as amostras foram transferidas para as peneiras de aço inox de abertura de malha 2,00mm (ABNT $\left.\mathrm{n}^{\circ} 10\right), 0,59 \mathrm{~mm}\left(\mathrm{ABNT} \mathrm{n}^{\mathbf{0}} 30\right)$ e $0,150 \mathrm{~mm}$ $\left(A B N T n^{\circ} 100\right)$, encaixadas uma sobre a outra, mantendo a de malha maior por cima.

Após a agitação dessas peneiras, por 5 minutos em agitador mecânico marca RETSCH AS200, amplitude 1,51 $\mathrm{mm} / \mathrm{g}$, as frações retidas em cada peneira foram retiradas e pesadas. A análise do carbonato foi realizada baseada no método da EMBRAPA (Silva, 2009). Foram pesadas 2,0 g da amostra de sedimento seco e adicionados $200 \mathrm{~mL}$ de água ultrapura obtida através de um sistema de purificação MILLI$\mathrm{Q}^{\circledR}$ Pluswater (Millipore Molsheim, França) e $50 \mathrm{~mL}$ ácido clorídrico $(\mathrm{HCl}) 0,5 \mathrm{~mol} \mathrm{~L}^{-1}$.

O sistema foi aquecido em placa por 5 minutos com posterior titulação, utilizando solução padrão de $\mathrm{NaOH} 0,25$ mol L-1 e fenolftaleína $1 \%$ como indicador. Para a obtenção da percentagem do carbono orgânico total (\% COT) foi pesado uma porção da amostra (10-12 mg) de sedimento em uma cápsula de estanho seguido de análise elementar, conforme aplicado por Costa et al., (2011), sendo determinado pelo analisador elementar Marca CHNS-O (Analyser Costech Instruments Modelo ECS 4010). O carbono orgânico total (COT) foi determinado em triplicatas com um desvio padrão relativo inferior a 1\% (Santos, 2012). A quantidade de MO, foi obtida pela multiplicação dos valores de COT pelo fator 1,724 , convertendo assim COT em MO (\% MO = \% COT $\mathrm{x}$ $1,724)$. Este cálculo foi realizado tendo como base o conceito de que a quantidade de carbono da MO é de $58 \%$ (Conceição et al., 1999).

O procedimento da extração parcial dos elementos metálicos foi realizado baseado no método 3050B EPA (EPA, 1996). Uma massa de $1,0 \mathrm{~g}$ de amostra de sedimento foi pesada em tubos de vidro aos quais foram adicionados $10 \mathrm{~mL}$ de ácido nítrico concentrado $\left(\mathrm{HNO}_{3}\right)$ e $5 \mathrm{~mL}$ de peróxido de hidrogênio $\left(\mathrm{H}_{2} \mathrm{O}_{2}\right) 30 \%\left(\mathrm{v} \mathrm{v}^{-1}\right)$ para posterior digestão em bloco digestor com refluxo utilizando dedo frio, a uma temperatura de $95^{\circ} \mathrm{C}$ por 3 horas, evitando assim a perda de analitos voláteis e excessiva evaporação dos ácidos durante o aquecimento (Ferreira et al., 2013).

Em seguida, foram adicionados $10 \mathrm{~mL}$ de $\mathrm{HCl} 12 \mathrm{~mol} \mathrm{~L}^{-1}$. Após o resfriamento do tubo, em temperatura ambiente, a solução foi transferida para tubos de centrífuga e o volume ajustado para $50 \mathrm{~mL}$, com água ultrapura.

A determinação dos metais foi por ICP OES, simultâneo com visão axial, para determinação dos elementos $\mathrm{Cu}, \mathrm{Ba}$, Mo, V, Fe, Ni e Mn nas seguintes linhas espectrais: Ba (455.403,II); $\mathrm{Cu} \quad(237.395, \mathrm{I}) ; \quad \mathrm{Fe} \quad(238.204, \mathrm{II}) ; \quad \mathrm{Mn}$ (257.610,II); Mo (202.032,); Ni (231.604,II); V (292.401,II). As amostras foram digeridas em triplicatas juntamente com as soluções do branco e o material de referência certificado (CRM) de sedimento estuarino, adquirido do National Institute of Standard and Technology - NIST 1646a.

Cada lote de amostras digeridas foi preparado com 3 brancos de digestão para verificar possíveis contaminações no procedimento de digestão. Foi preparada uma solução multielementar para determinação dos elementos traço com concentração de $50 \mathrm{mg} \mathrm{L}^{-1}$ a partir de soluções estoque monoelementares (Merck - Darmstadt, Alemanha). Foram 
preparados 09 padrões (concentração de 0,05 a $5 \mathrm{mg} \mathrm{L}^{-1}$ ). A Tabela 1 representa as condições de operação do ICP OES.

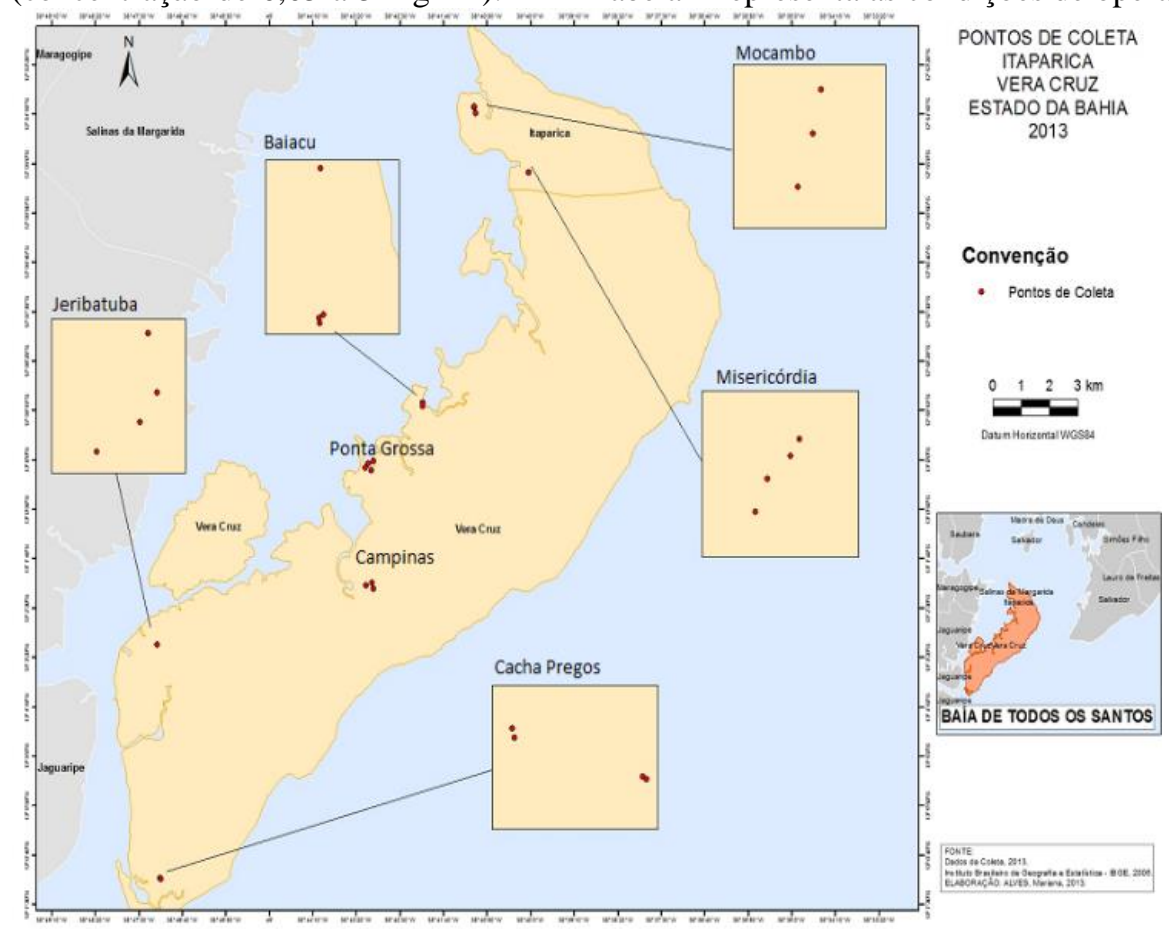

Figura 1. Localização dos pontos de amostragem na Ilha de Itaparica, Bahia

Tabela 1. Parâmetros do ICP OES empregados para a determinação dos elementos químicos nas amostras de sedimentos.

\begin{tabular}{|c|c|}
\hline Parâmetros & Valores \\
\hline Potência RF (kW) & 1.3 \\
\hline Vazão do gás de nebulização $\left(\mathrm{L} \min ^{-1}\right)$ & 0.70 \\
\hline Vazão do gás auxiliar ( $\left.\mathrm{L} \mathrm{min}^{-1}\right)$ & 1.5 \\
\hline Vazão do gás do plasma $\left(\mathrm{L} \min ^{-1}\right)$ & 15 \\
\hline Tempo de integração (s) & 1.0 \\
\hline Tempo de estabilização (s) & 15 \\
\hline Tempo de leitura (min) & 1 \\
\hline Replicatas & 3 \\
\hline Nebulizador & Concêntrico \\
\hline Câmara de Nebulização & Ciclônica \\
\hline
\end{tabular}

A exatidão do método foi verificada por meio de análises em triplicata do material de referência certificado de sedimento estuarino (NIST 1646a). Os valores de recuperação para as amostras de sedimentos do CRM variaram de $62 \%$ para o $\mathrm{Zn}$ a $87 \%$ para o $\mathrm{V}$.

Para avaliar a precisão do método utilizado, as amostras foram analisadas em triplicata, considerando-se os valores médios aceitáveis, sendo o desvio padrão relativo entre as réplicas inferior a $15 \%$. Os dados obtidos foram submetidos à análise estatística empregando Matriz de Correlação de Pearson (estatística descritiva) através do programa Microsoft Excel 2007, e a análise multivariada, Análise de Componentes Principais (PCA), utilizando o programa Statistica 8.0.

Os valores expressos para limite de deteç̧ão (LD) e de quantificação (LQ) foram calculados com base nas expressões: $\mathrm{LD}=3 s / \mathrm{S}$ e $\mathrm{LQ}=10 s / \mathrm{S}$, respectivamente, em que $\boldsymbol{s}$ é o desvio padrão referente a 10 medidas do branco e $\mathbf{S}$ é o coeficiente angular da curva de calibração (Templeton et al.,
2000). Os valores dos referidos limites de cada analito estão expressos na Tabela 2.

Tabela 2. Limite de Detecção (LD) e Limite de Quantificação (LQ) do $\mathrm{Fe}\left(\mu \mathrm{g} \mathrm{g}^{-1}\right.$ x 10000) e Ba, Cr, $\mathrm{Cu}, \mathrm{Mn}, \mathrm{Ni}, \mathrm{V}, \mathrm{Zn}$ e Mo $\left(\mu \mathrm{g} \mathrm{g}^{-1}\right)$ determinados nas amostras de sedimento de manguezal da Ilha de Itaparica $(\mathrm{n}=28)$.

\begin{tabular}{cll|rll}
\hline Metal & LD & LQ & Metal & LD & LQ \\
\hline $\mathrm{Ba}$ & 0,022 & 0,074 & $\mathrm{Ni}$ & 0,007 & 0,024 \\
$\mathrm{Cu}$ & 0,004 & 0,012 & $\mathrm{~V}$ & 0,003 & 0,010 \\
$\mathrm{Fe}$ & 0,278 & 0,928 & $\mathrm{Mo}$ & 0,025 & 0,082 \\
$\mathrm{Mn}$ & 0,002 & 0,006 & & & \\
\hline
\end{tabular}

\section{Resultados e Discussão}

As concentrações médias para os elementos determinados nas amostras de sedimentos estão apresentadas nas Tabelas 3 e 4. Os valores reportam maiores concentrações de Mn nas amostras de CP, MI, BA e PG comparando-se com outras amostras já pesquisadas nas mesmas regiões. Foram determinados maiores valores de concentrações do elemento $\mathrm{Ba}$ nas amostras de MI, CP e JE, além de $\mathrm{Cu}$ em amostras de $\mathrm{CP}$, assim como $\mathrm{V}$ e Mo nas amostras de $\mathrm{MI}$ em relação as outras regiões.

Estudos desenvolvidos em manguezais da Baía de Todos os Santos (BTS) e outras regiões mostraram a presença de metais nos sedimentos (Queiroz e Celino, 2008) e os resultados de $\mathrm{Fe}, \mathrm{Mn}$ e $\mathrm{Cu}$ obtidos em regiões da BTS e na Ilha de Itaparica demonstraram faixas de concentração superiores às encontradas neste estudo. Seguindo esta mesma linha de 
comparação com relação ao $\mathrm{Fe}, \mathrm{Mn}$ e $\mathrm{Cu}$, também foi encontrado em pesquisa realizada (Santos et al., 2017), teores desses metais em maiores concentrações que este trabalho. Foi detectado em todos os pontos de coleta, o Fe como elemento que apresentou as maiores concentrações. Resultados similares foram reportados por Andrade (2011), para amostras de sedimentos na mesma área de estudo, onde se observa que a maior retenção dos elementos na parte mais interna do rio, é provavelmente, devido a maior predominância de sedimentos com as frações mais finas (silte e argila).

Segundo Queiroz e Celino (2008) as localidades próximas a áreas de atividades petrolíferas (Madre de Deus) e locais sob a influência do Rio Subaé e atividades humanas na região de São Francisco do Conde na BTS justificam as concentrações maiores para os analitos aqui avaliados.

As maiores variações de MO nas regiões de Misericórdia
(Tabela 5), seguida por Campinas, pode ser justificado pela maior presença de vegetação do mangue que ficam superficialmente acumuladas nos sedimentos e sob condições anaeróbicas são decompostas formando o material orgânico. Por outro lado, nos povoados de MU e JE, as faixas destes teores foram encontradas em menores quantidades.

$\mathrm{Na}$ Tabela 5, estão indicados valores de frações granulométricas mais finas encontradas nas regiões JE, MI e MU e menor quantidade encontrada na região de Ponta Grossa.

Já as mais elevadas variações equivalentes a carbonato de cálcio adquiridas neste estudo foram observadas nas regiões de Cacha Pregos e Mocambo, que provavelmente provém de fragmentos de conchas de moluscos e algas calcárias presentes neste ambiente.

Tabela 3. Faixa de concentração e seus desvios padrão dos elementos químicos determinados nas amostras de sedimento de manguezal das regiões (Campinas (CA); Misericórdia (MI); Cacha Pregos (CP); Jeribatuba (JE)) da Ilha de Itaparica, Bahia $(\mathrm{n}=28)$.

\begin{tabular}{ccccc}
\hline Metal & CA & MI & CP & JE \\
\hline $\mathrm{Ba}$ & $0,62 \pm 0,02-3,94 \pm 1,51$ & $5,27 \pm 0,37-11,01 \pm 1,71$ & $2,71 \pm 0,38-9,77 \pm 1,42$ & $4,07 \pm 0,48-9,88 \pm 0,46$ \\
$\mathrm{Cu}$ & $0,87 \pm 0,21-1,12 \pm 1,66$ & $1,84 \pm 0,22-3,42 \pm 0,04$ & $0,60 \pm 0,18-20,38 \pm 6,55$ & $0,61 \pm 0,84-2,47 \pm 2,88$ \\
$\mathrm{Fe}$ & $0,04 \pm 0,00-0,19 \pm 0,05$ & $0,35 \pm 0,03-0,61 \pm 0,09$ & $0,10 \pm 0,02-0,47 \pm 0,04$ & $0,07 \pm 0,01-0,10 \pm 0,02$ \\
$\mathrm{Mn}$ & $2,07 \pm 0,20-8,60 \pm 2,57$ & $20,54 \pm 1,47-36,35 \pm 1,14$ & $7,78 \pm 0,35-26,75 \pm 2,51$ & $3,51 \pm 0,92-4,51$ a 0,68 \\
$\mathrm{Ni}$ & $0,41 \pm 0,17-1,60 \pm 0,36$ & $2,41 \pm 0,46-4,72 \pm 1,06$ & $1,41 \pm 0,25-3,62 \pm 2,51$ & $0,26 \pm 0,10-1,31 \pm 0,45$ \\
$\mathrm{~V}$ & $0,63 \pm 0,21-3,02 \pm 1,03$ & $5,26 \pm 0,23-10,55 \pm 1,81$ & $1,87 \pm 0,45-5,55 \pm 0,57$ & $1,37 \pm 0,43-1,65 \pm 0,37$ \\
$\mathrm{Mo}$ & $0,33 \pm 0,00-2,84 \pm 0,94$ & $4,50 \pm 0,49-10,77 \pm 1,82$ & $1,28 \pm 0,46-6,77 \pm 15,3$ & $0,72 \pm 0,06-1,02 \pm 0,22$ \\
\hline $\mathrm{Fe}\left(\mu \mathrm{g} \mathrm{g}^{-1} \times 10000\right) \mathrm{e} \mathrm{Ba}, \mathrm{Cr}, \mathrm{Cu}, \mathrm{Mn}, \mathrm{Ni}, \mathrm{V}, \mathrm{Zn} \mathrm{e} \mathrm{Mo}\left(\mu \mathrm{g} \mathrm{g}^{-1}\right)$. & & &
\end{tabular}

Tabela 4. Faixa de concentração e seus desvios padrão dos elementos químicos determinados nas amostras de sedimento de manguezal das regiões (Baiacu (BA); Mocambo (MU); Ponta Grossa (PG)) da Ilha de Itaparica, Bahia ( $\mathrm{n}=28)$.

\begin{tabular}{clll}
\hline Metal & \multicolumn{1}{c}{ BA } & \multicolumn{1}{c}{ MU } & \multicolumn{1}{c}{ PG } \\
\hline $\mathrm{Ba}$ & $3,28 \pm 0,26-8,74 \pm 1,75$ & $1,68 \pm 0,31-2,34 \pm 0,45$ & $4,83 \pm 1,10-8,63 \pm 1,21$ \\
$\mathrm{Cu}$ & $1,11 \pm 0,37-1,46 \pm 0,28$ & $0,36 \pm 0,00-0,56 \pm 0,11$ & $0,66 \pm 0,10-1,56 \pm 0,17$ \\
$\mathrm{Fe}$ & $0,17 \pm 0,02-0,39 \pm 0,04$ & $0,08 \pm 0,00-0,12 \pm 0,00$ & $0,12 \pm 0,02-0,33 \pm 0,03$ \\
$\mathrm{Mn}$ & $11,90 \pm 1,28-26,68 \pm 2,22$ & $10,55 \pm 0,24-11,82 \pm 1,46$ & $9,02 \pm 2,62-32,74 \pm 3,21$ \\
$\mathrm{Ni}$ & $1,31 \pm 0,42-2,85 \pm 0,49$ & $0,58 \pm 0,16-0,92 \pm 0,09$ & $0,92 \pm 0,32-1,61 \pm 0,45$ \\
$\mathrm{~V}$ & $2,37 \pm 0,29-6,05 \pm 0,79$ & $1,79 \pm 0,29-2,40 \pm 0,11$ & $2,41 \pm 0,69-6,00 \pm 0,88$ \\
$\mathrm{Mo}$ & $1,89 \pm 0,23-6,30 \pm 1,15$ & $0,93 \pm 0,14-1,35 \pm 0,29$ & $1,50 \pm 0,55-5,21 \pm 0,60$ \\
\hline $\mathrm{Fe}\left(\mu \mathrm{g} \mathrm{g} \mathrm{g}^{-1} \times 10000\right) \mathrm{e} \mathrm{Ba}, \mathrm{Cr}, \mathrm{Cu}, \mathrm{Mn}, \mathrm{Ni}, \mathrm{V}, \mathrm{Zn} \mathrm{e} \mathrm{Mo}\left(\mu \mathrm{g} \mathrm{g}^{-1}\right)$.
\end{tabular}

Análises estatísticas expressas na matriz de correlação de Pearson e na PCA poderão estabelecer relações entre as variáveis medidas e as concentrações de metais encontradas, além de propor semelhanças entre grupos das amostras de sedimentos de manguezais coletados na ilha de Itaparica. Segundo Boaventura (2011), a PCA além de mostrar similaridade no comportamento dos grupos estudados, a mesma realiza a redução dos dados originais para alguns fatores, chamados de Componentes Principais (PC).

Os coeficientes de correlação entre os elementos metálicos determinados e a quantidade de MO e carbonato estão expressos na Tabela 6. Neste estudo, foi observado forte correlação positiva entre os elementos metálicos $\mathrm{Ba}, \mathrm{Mo}, \mathrm{Fe}$,
$\mathrm{V}$, Ni e Mn, somente o $\mathrm{Cu}$ apresentou baixo coeficiente de correlação com os outros elementos estudados.

Há o destaque da correlação do Fe com os elementos analisados, que pode ser atribuído à co-precipitação destes metais com óxidos-hidróxidos de $\mathrm{Fe}$, sendo o $\mathrm{Fe}$ um importante suporte geoquímico dos metais supracitados (Marques et al., 2011).

Correlações positivas também foram observadas entre o $\mathrm{CaCO}_{3}$ e os metais $\mathrm{Ba}, \mathrm{Mo}, \mathrm{Fe}, \mathrm{Mn}, \mathrm{Ni}$ e V. Considerado como uma das principais fases ligantes de metais presentes nos sedimentos, o $\mathrm{CaCO}_{3}$ é classificado como suporte geoquímico na retenção de elementos metálicos, o que explica os elevados resultados de coeficientes na associação entre estes compostos 
de baixa solubilidade e os elementos metálicos estudados (Fagnani et al., 2011; Belo et al., 2010).

A MO apresentou forte correlação com o $\mathrm{Fe}$ e Mn, como pode ser observado na Tabela 6 . Além da matéria orgânica dissolvida ou particulada, os óxi-hidróxidos de ferro $(\mathrm{Fe})$ e de manganês (Mn), os carbonatos e os sulfetos são as principais fases ligantes de metais nos sedimentos, além da fração particulada mais fina, capaz de realizar adsorção portanto, quimicamente este fato pode ser justificado pela ação das bactérias redutoras de sulfato (USEPA, 2005).

Estes microrganismos na presença da MO agem transferindo elétrons, oxidando a MO que dispõe de sítios de troca superficiais e reduzindo o sulfato, o que forma sulfetos de ferro e manganês, conseqüentemente pode haver a presença destes metais em ambientes anaeróbicos que são caracterizados pela acumulação de MO (Fagnani et al., 2011).

Tabela 5. Porcentagens dos teores de matéria orgânica (\%MO), de silte+argila e Carbonato de Cálcio $\left(\% \mathrm{CaCO}_{3}\right)$ das amostras de sedimentos superficiais de manguezais da Ilha de Itaparica, Bahia

\begin{tabular}{cccc}
\hline Estação & $\% \mathrm{MO} *$ & $\%$ Silte + Argila* & $\% \mathrm{CaCO}_{3}$ \\
\hline CP & $0,19-0,94$ & $21,5-26,3$ & $2,41-4,59$ \\
BA & $0,27-0,98$ & $14,4-17,3$ & $0,72-1,16$ \\
JE & $0,12-0,43$ & $13,5-43,2$ & $0,57-1,11$ \\
UM & $0,10-0,17$ & $12,9-34,3$ & $4,00-7,96$ \\
MI & $0,97-3,26$ & $16,5-35,8$ & $0,73-0,81$ \\
PG & $0,19-0,69$ & $2,20-8,8$ & $0,62-1,01$ \\
CA & $0,50-1,59$ & $14,9-21,5$ & $0,00-0,68$
\end{tabular}

*Fonte: Santos, 2012
Além disso, uma correlação moderada $(0,300$ a 0,700$)$ entre a $\mathrm{MO}$ e os demais metais foi observada, provavelmente porque em ambientes com grandes quantidades de $\mathrm{MO}$, como manguezais, há uma maior tendência à imobilização de metais por interações químicas de superfícies (quelação), além de associações à rede molecular não superficial nas moléculas constituintes da matéria orgânica (Samadfam et al., 1996).

A análise estatística de componentes principais mostrou que a soma das duas primeiras componentes principais explicou $86,13 \%$ da variabilidade dos dados, sendo a primeira componente principal com maior contribuição explicando $77,07 \%$ da variância total dos dados (Figura 2).

Tabela 6. Matriz de correlação de Pearson dos elementos metálicos, Matéria Orgânica (MO) e Carbonato de Cálcio $\left(\mathrm{CaCO}_{3}\right)$ das amostras de sedimentos superficiais de manguezais da Ilha de Itaparica, Bahia $(\mathrm{p}<0,05)$.

\begin{tabular}{cccccccccc}
\hline Variável & $\mathrm{Ba}$ & $\mathrm{Mo}$ & $\mathrm{Cu}$ & $\mathrm{Fe}$ & $\mathrm{Mn}$ & $\mathrm{Ni}$ & $\mathrm{V}$ & $\mathrm{CaCO}_{3}$ & $\mathrm{MO}$ \\
\hline $\mathrm{Ba}$ & 1,00 & & & & & & & & \\
$\mathrm{Mo}$ & 0,70 & 1,00 & & & & & & & \\
$\mathrm{Cu}$ & 0,42 & 0,30 & 1,00 & & & & & & \\
$\mathrm{Fe}$ & 0,72 & 0,97 & 0,45 & 1,00 & & & & & \\
$\mathrm{Mn}$ & 0,69 & 0,93 & 0,34 & 0,93 & 1,00 & & & & \\
$\mathrm{Ni}$ & 0,62 & 0,89 & 0,35 & 0,93 & 0,82 & 1,00 & & & \\
$\mathrm{~V}$ & 0,70 & 0,99 & 0,34 & 0,98 & 0,91 & 0,93 & 1,00 & & \\
$\mathrm{CaCO}_{3}$ & 0,70 & 0,99 & 0,34 & 0,98 & 0,91 & 0,93 & 1,00 & 1,00 & \\
$\mathrm{MO}^{2}$ & 0,48 & 0,65 & 0,42 & 0,72 & 0,71 & 0,58 & 0,67 & 0,67 & 1,00 \\
\hline
\end{tabular}
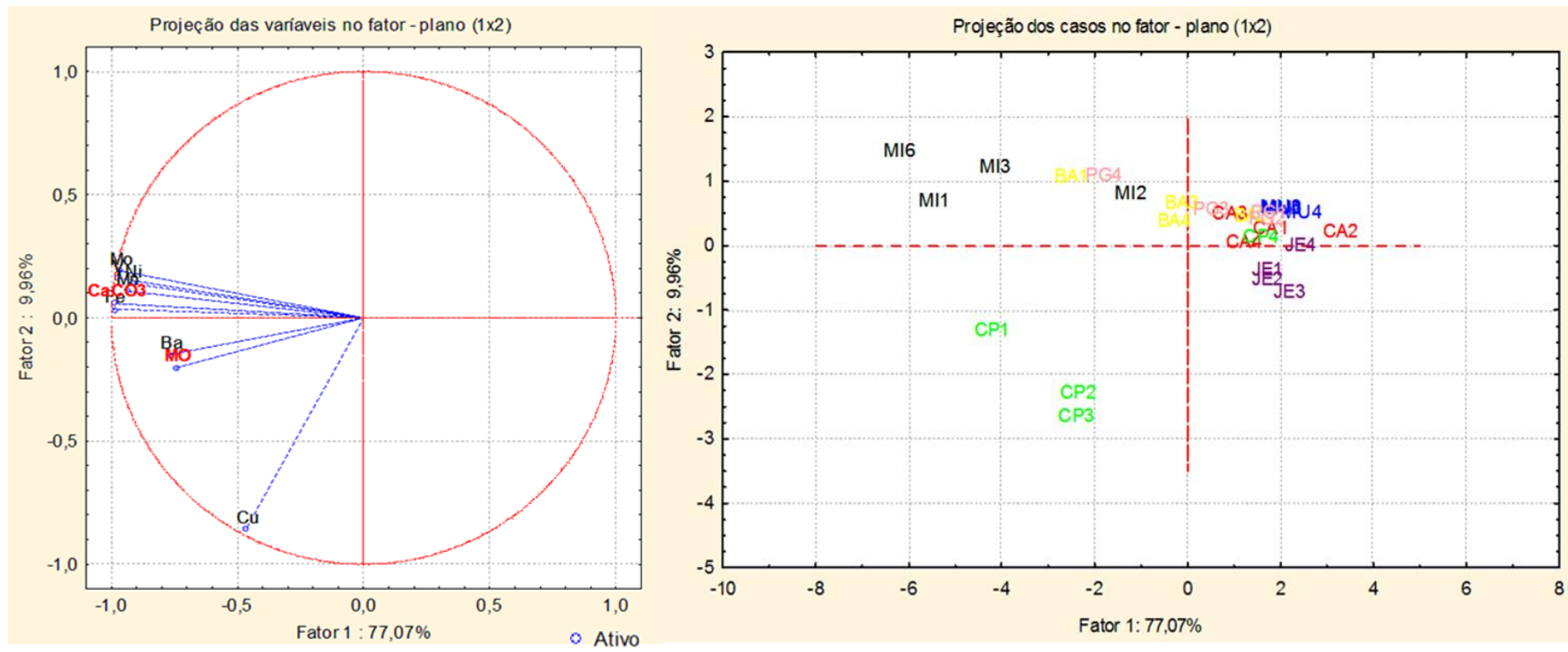

Figura 2. Gráficos da Análise de Componente Principal (PCA), referente aos pesos (esquerda) e aos escores (direita) das duas componentes principais $\left(\mathrm{PC}_{1} \times \mathrm{PC}_{2}\right)$

Reportando um efeito secundário na variação dos dados, o PC2 apresentou $9,96 \%$ dessa variação. São corroborados, na Figura 2, os elevados valores de coeficientes de correlação encontrados na Tabela 6, justificando a forte associação entre os metais $\mathrm{Mo}, \mathrm{Fe}, \mathrm{Mn}, \mathrm{Ni}, \mathrm{V}$ e $\mathrm{Ba}$ com os parâmetros avaliados $\left(\mathrm{CaCO}_{3}\right.$ e $\left.\mathrm{MO}\right)$.
O gráfico de escores das amostras analisadas, indicam tendência de separação das mesmas em função do local da coleta, principalmente nas amostras de Misericórdia e Cacha Pregos. Isso pode ser justificado pelas maiores quantidades de MO presentes nas amostras MI e $\mathrm{CaCO}_{3}$ nas amostras de $\mathrm{CP}$, as quais estão de acordo com os resultados na Figura 2. 
Também é observada uma tendência de separação nas amostras JE. A diferença da variação na quantidade de MO e as maiores concentrações de $\mathrm{Ba}$, indicadas nas Tabelas 3 e 4 aliada à elevada faixa de silte e argila (Tabela 5) encontrada neste local podem ter contribuído para essa tendência de separação das amostras desta região. A interação de grãos finos (silte e argila) com a MO em suspensão e a solubilidade dos metais em água intersticial causa a diminuição da energia do sistema (Dohierty, 2000). Como consequência, a sedimentação que contribui para a adsorção dos elementos químicos supracitados, pode tornar este ponto um reservatório de contaminantes.

\section{Conclusão}

A análise das amostras sedimentos coletadas nas regiões da Ilha de Itaparica evidenciou a presença de maiores concentrações de $\mathrm{Ba}, \mathrm{V}$, Mo e $\mathrm{Cu}$ principalmente nas regiões de Misericórdia, Cacha Pregos e Jeribatuba comparando-se com as demais regiões. As análises estatísticas empregadas permitiram discriminar as amostras em relação aos pontos de coleta, bem como identificar algumas relações existentes entre os dados obtidos dos elementos metálicos e as variáveis avaliadas. De acordo com os resultados registrados na matriz de correlação ficou evidente a interação entre os metais Ba, $\mathrm{Mo}, \mathrm{Fe}, \mathrm{V}$, Ni e $\mathrm{Mn}$ e o $\mathrm{CaCO}_{3}$ presente nas amostras de sedimento, além da forte relação entre a MO e os elementos $\mathrm{Fe}$ e Mn. Os resultados de PCA confirmaram os dados encontrados na matriz de correlação, além de indicarem correlação similar entre a MO e o Ba no gráfico de pesos.

Em relação à origem dos substratos coletados, foi mostrada no gráfico de escores uma predisposição de separação das amostras, o qual foi mais evidente para as regiões de Misericórdia, Cacha Pregos e Jeribatuba. Nestas regiões, as concentrações de alguns metais foram superiores em relação às demais, devido a associação com o $\mathrm{CaCO}_{3}$ e a MO e possivelmente pelas características granulométricas do sedimento obtidas em estudos preliminares, com maior fração de silte e argila, que possuem maior superfície de contato e capacidade de adsorção dos elementos metálicos.

\section{Agradecimentos}

Ao Grupo de Pesquisa em Química Analítica (GPQA/IQ/UFBA) pelo apoio durante as análises químicas.

\section{Referências}

Andrade, C.L.N. 2011. Biogeoquímica do ambiente estuarino do rio passa vaca em área urbana de Salvador, BA. Dissertação de Mestrado, Instituto de Geociências/Universidade Federal da Bahia, Salvador, Bahia. 140p.

Belo, A.; Quináia S.P.; Pletsch, A.L. 2010._Avaliação da contaminação de metais em sedimentos superficiais das praias do lago de Itaipu. Química Nova, 33(3): 613-617.

Boaventura, S. F. 2011. Distribuição de metais traço em superfície no município de Madre de Deus, Bahia. Dissertação de Mestrado em Geoquímica: Petróleo e Meio Ambiente/Universidade Federal da Bahia, Salvador, Bahia.

Carapeto, C. 2008. Poluição das águas: causas e efeitos. Lisboa: Universidade Aberta, 241p.

Castro, P; Huber, M.E. 2012. Biologia Marinha. 8a. ed. AMGH, Porto Alegre, 460p.

Conceição, M. da; Manzatto, C. V.; Araújo, W. S. de; Martin Neto, L.; Saab, S. da C.; Cunha, T. J. F. da; Freixo, A. A. 1999. EMBRAPA. Estudo comparativo de métodos de determinação do teor de Matéria Orgânica em solos orgânicos do estado do Rio de Janeiro. Ministério da
Agricultura e do Abastecimento. 3: 1-5.

Costa, A.B.; Novotny, E.H.; Bloise, A.C.; de Azevedo, E.R.; Bonagamba, T.J.; Zucchi, M.R.; Santos, V.L.C.S.; Azevedo, A.E.G. 2011. Characterization of organic matter in sediment cores of the Todos os Santos Bay, Bahia, Brazil, by elemental analysis and ${ }^{13} \mathrm{C}$ NMR. Marine Pollution Bulletin, 62(8): 1883-1890.

Dohierty, G.B.; Brunskill, G.J.; Ridd, M.J. 2000. Natural and enhanced concentrations of trace metals in sediments of Cleveland Bay, Great Barrier Reef Lagoon, Australia. Marine Pollution Bulletin. 41(7-12): 337344.

Dou, Y.; Li J.; Zhao J.; Hu B.; Yang S. 2013. Distribuition, enrichment and source of heavy metals in sufarce sediments of the eastern Beibu Bay, South China Sea. Marine Pollution Bulletin. 67(1-2): 137-145.

EPA-Environmental Protection Agency, 1996. Method 3050B-4: Acid digestion of sediments, sludges, and soils. Revision 2. CD-ROM.

Fagnani, E.; Guimarães, J.R.; Mozeto, A.A.; Fadini, P.S. 2011. Sulfetos volatilizáveis por acidificação e metais extraídos simultaneamente na avaliação de sedimentos de água doce. Quimica Nova, 34 (9): 1618-1628.

Ferreira, S.L.C.; Silva, L.O.B.; Santana, F.A.de; Junior, M.M.S.; Matos, G.D.; Santos, W.N.L. dos. 2013. A review of reflux systems using cold finger for sample preparation in the determination of volatile elements. Microchemical Journal, 106: 307-310.

Fortunato, J.M., 2009. Comportamento dos íons chumbo, cobre, níquel e zinco em área de manguezal associado ao antigo lixão no município de Santos SP. Dissertação de Mestrado em Recursos Minerais e Hidrogeologia/Universidade de São Paulo, São Paulo, São Paulo. 75p.

Gan, H.; Lin, J.; Liang, K.; Xia, Z. 2013. Selected trace metals (As, Cd and $\mathrm{Hg}$ ) distribution and contamination in the coastal wetland sediment of the northern Beibu Gulf, South China Sea. Marine Pollution Bulletin, 66 (12): $252-258$

Marques, J.S.J.; Rangel, T.P.; Brito, F.P.; Almeida, M.G.; Salomão, M.S.M.B.; Gobo, A.A.R.; Souza-Santos, L.P.; Araújo-Castro, C.M.V.; Costa, M.F.; Rezende, C.E. 2011. Geoquímica de Metais em Sedimentos da Zona Estuarina do Complexo Industrial Porto de Suape, PE-Brasil. Revista da Gestão Costeira Integrada, 11(4): 379-387.

Martins, S. S. S.; Silva, M. P. M.; Azevedo, O. Silva, V. P. 2015. Produção de Petróleo e Impactos Ambientais. Holos, 31(6): 54-76.

Queiróz, A.F. de S., Celino, J.J., 2008. Manguezais e ecossistemas estuarinos na Baía de Todos os Santos. In: Queiroz, A.F. de S.; Celino, J.J. Avaliação de ambientes na Baía de Todos os Santos: aspectos geoquímicos, geofísicos e biológicos. $1^{\mathrm{a}}$ ed. Salvador: EDUFBA, 1, 39-58. 300p.

Samadfam, M., Niitsu, Y., Sato,S., Ohashi, H., 1996. Complexation thermodynamics of $\mathrm{Sr}$ (II) and humic acid. Radiochimica Acta, 73(4): 211-216.

Santos E. C., 2012. Hidrocarbonetos Policíclicos Aromáticos (HPA's) eSaturados em sedimentos superficiais de manguezais da ilha de Itaparica, Bahia, Brasil. Dissertação de Mestrado em Geoquímica: Petróleo e Meio Ambiente/Universidade Federal da Bahia, Salvador, Bahia. 190 p.

Santos, L.M.M.; Souza, R.C.; Anunciação, D.S.; Viana, Z.C.V.; Santos, V.L.C.S. 2017. Análise de metais em sedimentos de manguezal do Rio Passa Vaca, Salvador, Brasil. Acta Brasiliensis, 1(2):1-7.

Silva, F. C. da. 2009. Manual de análises químicas de solos, plantas e fertilizantes. Embrapa Informação Tecnológica, Brasília, DF. 2 ${ }^{\mathrm{a} \cdot e d .}$ 627p.

Templeton, d. M.; Ariese, F.; Cornelis, R.; Danielsson, L-G., Muntau, H., Leeuwen, H.P.V., Lobinski, R. 2000. IUPAC. Guidelines for terms related to chemical speciation and fraction of elements. Definitions, structural aspects and methodological approaches. Pure and Applied Chemistry, 72(8): 1453-1470.

USEPA - United States Environmental Protection Agency; Procedures for the derivation of equilibrium partitioning sediment benchmarks (ESBs) for the protection of benthic organisms: Metal mixtures (cadmium copper, lead nickel, silver, and zinc), EPA-600-R-02-011, Office of Research and Development: Washington DC, 2005. 